\section{ANÁLISE MICROBIANA E DO TEOR DE FLÚOR ADICIONADO À ÁGUA DE ABASTECIMENTO EM UM ESTADO DO NORDESTE DO BRASIL}

\author{
Analysis of bacterial and fluoride content added to water supply \\ in Northeastern Brazil
}

\author{
Análisis microbiano y de la concentración del flúor añadido \\ al agua del abastecimiento de un estado del noreste de Brasil
}

Artigo Original

\section{RESUMO}

Objetivo: Analisar os teores de íons de fluoreto e o padrão microbiológico encontrado nas águas de abastecimento público dos municípios alagoanos que fluoretam suas águas. Métodos: Estudo transversal descritivo realizado no período de 2012 a 2014, no qual se analisaram todos os laudos emitidos pelo Laboratório Central de Saúde Pública de Alagoas (LACEN-AL) com os resultados da análise da qualidade da água para consumo humano dos onze municípios alagoanos que fluoretaram suas águas, totalizando 3.089 laudos. Avaliouse o número total de amostras enviadas para análise da qualidade da água, a quantidade de amostras em que foi solicitada a análise de fluoreto, teores e variações dos íons de fluoreto e o padrão microbiológico das amostras fluoretadas. Realizou-se a análise descritiva dos dados, obtendo-se as frequências absolutas e relativas percentuais. Resultados: Foram encontradas $429(83,9 \%)$ amostras coletadas no intervalo $0,0-0,5 \mathrm{mgF} / \mathrm{L}$, número considerado abaixo do recomendável, além de grande variação na concentração dos íons de fluoreto e 128 $(26,3 \%)$ amostras de água fluoretada fora dos padrões de potabilidade. Conclusão: Os dados mostraram uma grande variação e um alto percentual de amostras com baixas concentrações de fluoreto, assim como a necessidade de melhoria da qualidade da água ofertada, de modo a garantir para a população o acesso contínuo à água potável.

Descritores: Cárie Dentaria; Fluoretação; Qualidade da Água.

\section{ABSTRACT}

Objective: To assess the levels of fluoride ions and microbiological standard present in the public water supply of cities that fluoridate their water in the state of Alagoas. Methods: Descriptive cross sectional study conducted in the period 2012-2014 to analyze all reports issued by the Public Health Central Laboratory of Alagoas (Laboratorio Central de Saúde Pública de Alagoas - LACEN-AL) with the results of drinking-water analysis of the eleven cities in Alagoas that fluoridated their water - a total of 3,089 reports. We assessed the total number of samples sent for analysis of water quality, the number of samples for analysis of fluoride, levels and variation of fluoride ions, and the microbiological standard of fluoride samples. Data underwent descriptive analysis with absolute frequencies and percentages. Results: 429 (83.9\%) of the samples collected were in the range 0.0-0.5 mgF/L, which is considered below the recommended levels, and there was a great variation in the concentration of fluoride ions; 128 (26.3\%) fluoridated water samples were outside the potability standards. Conclusion: Data showed a great variation and a high percentage of samples with low concentrations of fluoride as well as the need to improve the quality of water provided in order to guarantee a continued access to drinking-water.
1) Laboratório Central de Saúde Pública Dr. Aristeu Lopes - LACEN - Maceió (AL) - Brasil

2) Universidade Federal de Alagoas UFAL- Maceió (AL) - Brasil

3) Universidade Federal do Rio de Janeiro UFRJ - Rio de Janeiro (RJ) - Brasil
Recebido em: 13/03/2015 Revisado em: 22/04/2015 Aceito em: 13/05/2015 


\section{RESUMEN}

Objetivo: Analizar las concentraciones de los iones de fluoruro y el patrón microbiológico de las aguas del abastecimiento público de los municipios de Alagoas que añaden el flúor en sus aguas. Métodos: Estudio transversal descriptivo realizado entre 2012 y 2014 en el cual se analizó todos los laudos emitidos por el Laboratorio Central de Salud Pública de Alagoas (LACEN-AL) con los resultados del análisis de la calidad del agua para el consumo humano de los once municipios de Alagoas que añadieron el flúor en sus aguas en un total de 3.089 laudos. Se evaluó el número total de muestras enviadas para el análisis de la calidad del agua, la cantidad de muestras de las cuales fueron solicitadas el análisis del fluoruro, las concentraciones y variaciones de los iones de fluoruro y el patrón microbiológico de las muestras con flúor. Se realizó el análisis descriptivo de los datos obteniéndose las frecuencias absolutas y las relativas porcentuales. Resultados: Fueron encontradas 429 (83,9\%) muestras recogidas en el intervalo de 0,0-0,5 $\mathrm{mgF} / \mathrm{L}$, número abajo del recomendable, además de una gran variación de la concentración de los iones de fluoruro y 128 (26,3\%) muestras del agua con flúor fuera de los patrones de potabilidad. Conclusión: Los datos muestran una gran variación y un elevado porcentual de muestras con bajas concentraciones de flúor asi como la necesidad de mejoría en la calidad del agua ofrecida de manera a garantizar la población el acceso continuo de agua potable.

Descriptores: Caries Dental; Fluorucación; Calidad del Agua.

\section{INTRODUÇÃO}

A água é essencial à vida, por isso, para livrá-la de qualquer substância ofensiva à saúde, buscou-se meios para torná-la potável ${ }^{(1)}$. As doenças diarreicas estão diretamente relacionadas às condições de abastecimento hídrico, saneamento básico e higiene, que, aliadas à falta de conhecimento da população, aumentam o aparecimento de doenças transmitidas pela água, principalmente em crianças e jovens, o que torna a avaliação da potabilidade da água e as condições higiênico-sanitárias vitais ao bem-estar da população ${ }^{(2-4)}$.

No Brasil, a água considerada com qualidade deve estar legalmente de acordo com a Portaria $n^{\circ} 2914$, de 12 de dezembro de 2011, do Ministério da Saúde ${ }^{(5)}$, que dispõe sobre os procedimentos de controle e vigilância da qualidade da água para consumo humano e seu padrão de potabilidade. São também estabelecidos nessa portaria os valores máximos permitidos (VMP) para os parâmetros físico-químicos e microbiológicos ${ }^{(1,6)}$.

O padrão de potabilidade para a água para consumo humano, sob o ponto de vista microbiológico, deve apresentar ausência de coliformes totais e de Escherichia coli (E. coli) em amostras com $100 \mathrm{~mL}$ de água ${ }^{(5)}$. O grupo dos coliformes totais inclui bactérias que podem ocorrer naturalmente no solo, na água e em plantas. A Escherichia coli, por outro lado, é apenas de origem fecal, sendo utilizada como o indicador de maior precisão desse tipo de contaminação ${ }^{(7)}$

A concentração de fluoreto é outro parâmetro importante na avaliação da qualidade nas águas de consumo. O Programa Brasil Sorridente ${ }^{(8)}$ visa melhorar as condições de saúde bucal no Brasil, ampliando a cobertura de água fluoretada de abastecimento ${ }^{(9)}$. No entanto, para que a fluoretação das águas seja efetiva em relação à prevenção da cárie dentária, é imprescindível que a adição dos íons de fluoreto seja feita de forma contínua e observando os teores adequados para cada localidade $\mathrm{e}^{(10,11)}$.

Devido a essa particularidade, torna-se imprescindível um controle rigoroso. Assim, os responsáveis pelo fornecimento da água têm a responsabilidade de manter um controle operacional preventivo e permanente da potabilidade dessa água, que deve ser feito desde a sua saída da Estação de Tratamento de Água (ETA) até a sua entrada nas ligações domiciliares ${ }^{(12)}$. Na área da vigilância, é necessário fazer o heterocontrole, que consiste na realização de ações de controle e vigilância por uma instituição diferente daquela responsável pela execução do abastecimento, visando à manutenção do padrão de qualidade $^{(13)}$.

Para o fluoreto, o valor máximo permitido é de 1,5 mgF/L desde o ano 2000, quando da publicação da Portaria $n^{\circ} 1.469 / 2000$. Esse valor foi mantido na Portaria $n^{o} 518 / 2004^{(10)}$ e na Portaria $n^{o} 2.914 / 2011^{(5)}$. Porém, sob a ótica da vigilância em saúde quanto à avaliação da fluoretação da água no Brasil, o flúor em níveis inferiores a 0,6 ou superiores a $0,8 \mathrm{mg}$ de $\mathrm{F} / \mathrm{L}$ é considerado fora dos limites aceitáveis ${ }^{(14)}$.

A temperatura é um importante fator ambiental que deve ser considerado ao se calcular o teor de fluoreto necessário na água de abastecimento de uma determinada localidade, pois está diretamente ligado ao consumo de água ${ }^{(15,16)}$. A ingestão contínua de fluoretos em níveis acima do recomendado pode ocasionar o aparecimento de fluorose dental $^{(15,17)}$.

O estado de Alagoas está localizado no leste da região Nordeste do Brasil e, segundo dados do Instituto Brasileiro de Geografia e Estatística (IBGE) ${ }^{(18)}$, possuía uma população de 3.120.922 habitantes em 2010 e uma densidade populacional de 112,33 hab/ $\mathrm{km}^{2}$, obtendo em 2013 o Índice de Desenvolvimento Humano (IDH) de $0,631^{(19)}$. É um estado cujas condições sociais, econômicas e de acesso à atenção à saúde apresentam grandes desigualdades ${ }^{(20,21)}$. Maceió é uma das poucas capitais brasileiras que não realiza a fluoretação das águas de abastecimentos públicos, e dentre os 102 municípios alagoanos, apenas onze adicionam flúor a suas águas ${ }^{(22,23)}$. 
A análise microbiológica é um requisito importante para assegurar a potabilidade da água de consumo, e a fluoretação, como um método de grande abrangência no controle da cárie dentária, traz no cerne um grande potencial de inclusão social que ajudaria a diminuir essas diferenças. Em vista disso, o objetivo do presente estudo foi analisar os teores de íons de fluoreto e o padrão microbiológico encontrados na água de abastecimento público dos municípios alagoanos que fluoretam suas águas.

\section{MÉTODOS}

Trata-se de um estudo transversal descritivo, no qual foram analisados laudos emitidos pelo Laboratório Central de Saúde Pública de Alagoas (LACEN-AL) com os resultados das amostras de água coletadas pela vigilância em saúde ambiental das secretarias da saúde dos municípios alagoanos que adicionam flúor à água de abastecimento público.

Analisaram-se os laudos do período compreendido entre 2012-2014, expedidos para os onze municípios que fluoretam a água de abastecimento. A eleição inicial do período deveu-se ao fato de que, com a entrada em vigor da Portaria 2.914/11(5), passou-se a não ser mais necessário, para a empresa responsável pela fluoretação, realizar a análise dos teores de fluoreto na rede de distribuição.

Os laudos encontram-se arquivados em um banco de dados eletrônico. Após autorização do Setor de Análise de Projetos do LACEN-AL para a realização da presente pesquisa, obtiveram-se um cadastro de usuário e a liberação de uma senha, requisitos necessários para acessar o sistema.

A coleta dos dados ocorreu entre os meses de maio de 2014 e janeiro de 2015, no período vespertino, por um único examinador devidamente calibrado, que preencheu inicialmente uma planilha Excel com os seguintes dados: número do processo, data da coleta, quantidade de amostras enviadas para a análise da qualidade da água para consumo humano e quantidade de amostras solicitadas para a análise dos íons de fluoreto. Posteriormente, esses dados foram agrupados por mês de envio. Em outra planilha Excel foram registrados os dados das amostras fluoretadas: número da amostra, data da coleta, teor de flúor e os dados do padrão microbiológico.

A responsabilidade de coletar as amostras para a análise da qualidade da água, enviá-las ao LACEN-AL e especificar as amostras que serão submetidas à análise dos íons de fluoreto é da vigilância em saúde ambiental municipal, o que faz com que a periodicidade do envio e a quantidade de amostras seja diferente de município para município.

Considerou-se no presente estudo como abaixo do recomendável os teores de fluoreto dentro do intervalo de 0,0 a $0,5 \mathrm{mgF} / \mathrm{L}$, como satisfatórios os teores dentro do intervalo 0,6 a $0,8 \mathrm{mgF} / \mathrm{L}$ e acima do recomendável qualquer teor acima de $0,8 \mathrm{mgF} / \mathrm{L}^{(14,24,25)}$. Com relação ao padrão microbiológico, avaliou-se a presença ou ausência de coliformes totais e de Escherichia coli em amostras com $100 \mathrm{~mL}$ de água ${ }^{(5)}$.

Mantiveram-se os nomes dos municípios em sigilo por questões éticas, sendo codificados em letras de A-K.

Os resultados foram tabulados e analisados pela estatística descritiva, através da qual se obtiveram as frequências absolutas e relativas percentuais.

\section{RESULTADOS}

A Tabela I apresenta o total de amostras enviadas pelas vigilâncias em saúde ambiental municipais para a análise da qualidade da água para consumo humano e o total de

Tabela I - Quantidade de amostras enviadas para análise da qualidade de água para consumo humano e para análise dos teores de íons de fluoreto. Maceió-AL, 2012-2014.

\begin{tabular}{ccccccc}
\hline & \multicolumn{2}{c}{$\mathbf{2 0 1 2}$} & \multicolumn{2}{c}{$\mathbf{2 0 1 3}$} & \multicolumn{2}{c}{$\mathbf{2 0 1 4}$} \\
\cline { 2 - 7 } Município & ACH & Fluoreto & ACH & Fluoreto & ACH & Fluoreto \\
\hline A & 121 & 06 & 170 & 15 & 165 & 25 \\
B & 215 & 61 & 175 & 38 & 130 & 37 \\
C & 42 & 05 & 59 & 0 & 93 & 0 \\
D & 150 & 50 & 160 & 52 & 170 & 60 \\
E & 139 & 16 & 14 & 01 & 115 & 17 \\
F & 111 & 0 & 27 & 0 & 10 & 0 \\
G & 60 & 20 & 40 & 12 & 50 & 16 \\
H & 90 & 06 & 120 & 10 & 85 & 03 \\
I & 31 & 03 & 44 & 0 & 40 & 02 \\
J & 74 & 06 & 95 & 21 & 50 & 14 \\
K & 100 & 15 & 44 & 0 & 100 & 0 \\
\hline TOTAL & $\mathbf{1 1 3 3}$ & $\mathbf{1 8 8}$ & $\mathbf{9 4 8}$ & 149 & 1008 & 174 \\
\hline
\end{tabular}

$\mathrm{ACH}=$ água para consumo humano. 
amostras em que se solicitou a análise dos íons de fluoreto nos onze municípios pesquisados. Enviou-se um total de 3.089 amostras para análise da água para consumo humano, sendo 1.133 amostras no ano de 2012, 948 amostras no ano de 2013 e 1.008 amostras no ano de 2014.

Constatou-se que, em um pequeno percentual das amostras - 16,5\% $(\mathrm{n}=511)$, foi solicitada a análise dos teores de íons de fluoreto. Observou-se também que entre os anos de 2012 e 2013 houve uma diminuição tanto no número total anual de amostras coletadas para análise da qualidade da água, quanto no número de amostras em que foi solicitada a análise dos íons de fluoreto. Contudo, entre os anos de 2013 e 2014, houve um aumento, sem, no entanto, ultrapassar os valores conseguidos no ano de 2012.
Os municípios de $\mathrm{C}$ e $\mathrm{K}$ não solicitaram análise dos íons de fluoreto nos anos de 2013 e 2014, e o município F não solicitou nos três anos pesquisados, apesar de esses municípios terem enviado amostras para a análise da qualidade de água para consumo humano.

No que diz respeito à Tabela II, ela retrata informações sobre a variação e os teores encontrados para os íons de fluoreto. Verificou-se que aproximadamente em $83 \%$ $(n=156), 88,6 \%(n=132)$ e $81 \%(n=141)$ das amostras analisadas nos anos de 2012, 2013 e 2014, respectivamente, houve teores de fluoreto na faixa considerada abaixo do recomendável $(0,0-0,5 \mathrm{mgF} / \mathrm{L})$.

Tabela II - Variação e teores dos íons fluoreto. Maceió-AL, 2012-2014.

\begin{tabular}{|c|c|c|c|c|c|c|c|c|c|c|c|c|}
\hline \multirow[b]{3}{*}{ Mun } & \multicolumn{4}{|c|}{2012} & \multicolumn{4}{|c|}{2013} & \multicolumn{4}{|c|}{2014} \\
\hline & \multicolumn{12}{|c|}{ Teores de fluoreto $(\mathrm{mg} \mathrm{F} / \mathrm{L})$} \\
\hline & $0,0 \mathrm{a}$ & $0,6 \mathrm{a}$ & $>0,8$ & Var & $0,0 \mathrm{a}$ & $0,6 \mathrm{a}$ & $>0,8$ & Var & $0,0 \mathrm{a}$ & $0,6 \mathrm{a}$ & $>$ & Var \\
\hline A & 6 & 0 & 0 & $0,0-0,3$ & 15 & 0 & 0 & $0,0-0,5$ & 25 & 0 & 0 & $0,0-0,3$ \\
\hline B & 47 & 7 & 7 & $0,0-2,2$ & 31 & 3 & 4 & $0,0-1,5$ & 33 & 2 & 1 & $0,0-1,0$ \\
\hline $\mathrm{C}$ & 5 & 0 & 0 & $0,1-0,5$ & 0 & 0 & 0 & NA & 0 & 0 & 0 & NA \\
\hline $\mathrm{D}$ & 48 & 0 & 2 & $0,0-2,0$ & 52 & 0 & 0 & $0,2-0,5$ & 56 & 2 & 2 & $0,0-1,0$ \\
\hline $\mathrm{E}$ & 16 & 0 & 0 & $0,0-0,5$ & 1 & 0 & 0 & 0,1 & 9 & 7 & 1 & $0,0-1,2$ \\
\hline $\mathrm{F}$ & 0 & 0 & 0 & NA & 0 & 0 & 0 & NA & 0 & 0 & 0 & NA \\
\hline G & 9 & 6 & 5 & $0,0-1,2$ & 8 & 2 & 2 & $0,0-1,2$ & 9 & 4 & 4 & $0,0-1,5$ \\
\hline $\mathrm{H}$ & 6 & 0 & 0 & $0,0-0,3$ & 10 & 0 & 0 & $0,0-0,2$ & 3 & 0 & 0 & $0,0-0,0$ \\
\hline I & 0 & 2 & 1 & $0,7-0,9$ & 0 & 0 & 0 & NA & 2 & 0 & 0 & $0,2-0,4$ \\
\hline J & 5 & 1 & 0 & $0,1-0,7$ & 15 & 6 & 0 & $0,1-0,7$ & 4 & 10 & 0 & $0,3-0,8$ \\
\hline K & 14 & 1 & 0 & $0,1-0,6$ & 0 & 0 & 0 & NA & 0 & 0 & 0 & NA \\
\hline Total & 156 & 17 & 15 & & 132 & 11 & 6 & & 141 & 25 & 8 & \\
\hline
\end{tabular}

Mun: município; Var: variação; NA: não se aplica

Tabela III - Quantidade de amostras de água fluoretada em desacordo com o padrão de potabilidade. Maceió-AL, $2012-2014$.

\begin{tabular}{|c|c|c|c|c|c|c|c|c|c|}
\hline \multirow[b]{2}{*}{ Mun } & \multicolumn{3}{|c|}{2012} & \multicolumn{3}{|c|}{2013} & \multicolumn{3}{|c|}{2014} \\
\hline & CT & $\mathbf{E C}$ & SS & CT & EC & SS & CT & EC & SS \\
\hline $\mathrm{A}$ & 3 & 1 & 0 & 11 & 4 & 0 & 16 & 6 & 0 \\
\hline B & 11 & 0 & 0 & 10 & 0 & 0 & 11 & 0 & 0 \\
\hline C & 1 & 1 & 0 & NA & NA & NA & NA & NA & NA \\
\hline D & 16 & 4 & 2 & 10 & 1 & 9 & 7 & 2 & 7 \\
\hline E & 4 & 1 & 0 & 0 & 0 & 0 & 3 & 2 & 0 \\
\hline $\mathrm{F}$ & NA & NA & NA & NA & NA & NA & NA & NA & NA \\
\hline G & 1 & 0 & 2 & 0 & 0 & 0 & 1 & 1 & 2 \\
\hline $\mathrm{H}$ & 1 & 0 & 1 & 3 & 1 & 0 & 0 & 0 & 0 \\
\hline I & 1 & 0 & 0 & NA & NA & NA & 1 & 1 & 0 \\
\hline $\mathrm{J}$ & 2 & 1 & 0 & 1 & 0 & 2 & 4 & 0 & 0 \\
\hline K & 10 & 2 & 0 & NA & NA & NA & NA & NA & NA \\
\hline Total & 50 & 10 & 5 & 35 & 6 & 11 & 43 & 12 & 9 \\
\hline
\end{tabular}

$\mathrm{NA}=$ não se aplica; $\mathrm{CT}=$ coliformes totais; $\mathrm{EC}=$ Escherichia coli; $\mathrm{SS}=$ sem solicitação; Mun: município 
O município B apresentou a maior variação nos teores de fluoreto durante os três anos pesquisados $(0,0-2,2$ (2012), 0,0-1,5 (2013), 0,0-1,0 (2014)), seguido pelo município G.

A Tabela III apresenta o resultado de amostras fluoretadas fora dos padrões de potabilidade recomendado pelo Ministério da Saúde, que preconiza a ausência de coliformes totais e de Escherichia coli em amostras com $100 \mathrm{~mL}$ de água ${ }^{(5)}$.

Não foi requerida a análise microbiológica em cinco amostras enviadas para análise dos íons de fluoreto no ano de 2012, em onze no ano de 2013 e em nove no ano de 2014. Entretanto, observou-se que mais de $20 \%$ $(\mathrm{n}=128)$ das amostras coletadas no período 2012-2014, nos onze municípios estudados, estavam fora do padrão microbiológico recomendado pela Portaria $\mathrm{n}^{\circ} 2.914$ do Ministério da Saúde ${ }^{(5)}$.

\section{DISCUSSÃO}

Com a entrada em vigor da Portaria $\mathrm{n}^{\circ} 2.914$ do Ministério da Saúde, em 12 de dezembro de 2011(5), passou a não ser mais necessário para a empresa responsável pela fluoretação realizar a análise dos teores de fluoreto na rede de distribuição, fazendo do controle realizado pelas vigilâncias em saúde municipais (heterocontrole) uma ferramenta valiosa para assegurar a qualidade da água fornecida ao consumidor.

Adiretriz nacional do plano de amostragem da vigilância ambiental em saúde relacionada à qualidade da água para consumo humano ${ }^{(26)}$ estabeleceu que o número mínimo de amostras a serem coletadas para implementação do plano de amostragem para a vigilância da qualidade da água para consumo humano deveria ser calculado de acordo com a população total do município, incluindo os diversos tipos de abastecimento de água. Assim, municípios com população menor ou igual a 50.000 habitantes deveriam realizar o mínimo de cinco amostras mensais para a análise dos teores dos íons de fluoreto, e municípios cuja população estivesse na faixa entre 50.001 a 100.000 habitantes deveriam realizar o mínimo de dez amostras mensais. Em 2014, uma nova diretriz foi publicada ${ }^{(27)}$, baixando a quantidade mínima de dez para sete amostras a serem coletadas nas localidades com população entre 50.001 e 100.000 habitantes.

Em 2010, os municípios G, H e J possuíam uma população de aproximadamente $60.389,54.591$ e 62.401 habitantes, respectivamente ${ }^{(16)}$, estando situados na faixa populacional de 50.001 a 100.000 habitantes; portanto, de acordo com as diretrizes citadas anteriormente, deveriam realizar o mínimo de dez amostras mensais nos anos de 2012 e 2013 e sete no ano de 2014. Já os demais municípios pesquisados no presente estudo possuíam uma população menor que 50.000 habitantes e deveriam realizar o mínimo de cinco amostras por mês ${ }^{(26,27)}$. Assim, apenas o município B, em 2012, e o município de D, em 2014, realizaram a avaliação da quantidade mínima de amostras exigidas no plano de amostragem; no ano de 2013, nenhum município atingiu a quantidade mínima estabelecida.

A água é o compartimento ambiental mais diretamente afetado pelas mudanças climáticas ${ }^{(26)}$. Em países tropicais com temperaturas máximas superiores a $27^{\circ} \mathrm{C}$, o mais adequado consistiria em utilizar concentrações de fluoreto entre $0,6-0,7 \mathrm{mgF} / \mathrm{L}^{(27)}$. Um estudo publicado em 2011, o qual levou em consideração as médias das temperaturas máximas diárias, definiu que a concentração ideal de fluoreto a ser adicionada à água de Maceió seria de 0,678 $\mathrm{mgF} / \mathrm{L}^{(28)}$. No presente estudo, o maior o percentual de amostras se concentrou no intervalo de $0,0-0,5 \mathrm{mgF} / \mathrm{L}$, considerado abaixo do recomendável ${ }^{(14,24,25)}$. Esses dados são compatíveis com o estudo realizado no Maranhão, segundo o qual $53,57 \%$ das amostras apresentaram concentrações inadequadas para íons de fluoreto ${ }^{(29)}$.

$\mathrm{Na}$ presente pesquisa, encontrou-se uma grande variação da concentração dos íons de fluoreto nas amostras analisadas, sendo o maior intervalo registrado de 0,0 a 2,2 $\mathrm{mgF} / \mathrm{L}$. Dados similares foram encontrados no município de Chapecó, em Santa Catarina ${ }^{(30)}$; por outro lado, no município de Canoas, Rio Grande do $\mathrm{Sul}^{(31)}$, houve uma menor variação, ficando a concentração de fluoreto dentro do intervalo de 0,1 a $1,6 \mathrm{mg} \mathrm{F} / \mathrm{L}$

Os resultados do atual estudo demonstraram que mais de $70 \%(\mathrm{n}=486)$ das amostras fluoretadas pesquisadas estavam dentro do padrão microbiológico exigido pela Portaria $n^{\circ} 2914 / 11$. Entretanto, os percentuais encontrados com presença de coliformes totais ou coliformes totais e E. coli, por exigência Ministério da Saúde, não deveriam existir em uma água considerada tratada e fluoretada ${ }^{(5)}$.

Os resultados encontrados na atual pesquisa refletem parcialmente a situação abordada, pois se deve levar em consideração as limitações de um estudo transversal, que realiza apenas um diagnóstico da situação proposta. Assim, sugere-se que novos estudos epidemiológicos sejam executados, de modo a aprofundar o tema, para que se possa discriminar e correlacionar de forma mais precisa as relações existentes com os achados encontrados no presente estudo. Porém, diante dos dados apresentados, torna-se necessária a implementação de medidas com o objetivo de fortalecer as ações preventivas e de heterocontrole, que não devem ser feitas tão somente por ações realizadas pela administração pública, mas através de um engajamento com diversos segmentos da sociedade, formando um consórcio social a fim de garantir à população o acesso contínuo a uma água potável e com teores adequados de fluoreto. 


\section{CONCLUSÃO}

Os dados mostraram uma grande variação e um alto percentual de amostras com baixas concentrações de fluoreto, o que pode ter propiciado a ineficácia da medida de prevenção da cárie dental para a população dos municípios estudados. Ficou evidenciada, também, a necessidade de melhoria da qualidade da água ofertada.

\section{AGRADECIMENTOS} Rabelo.

Os autores agradecem à Dra. Telma Pinheiro e à Gyulia

\section{REFERÊNCIAS}

1. Maciel AC, Yonekubo F, Oliveira Filho PC, Rios EM. Gerenciamento espacial do monitoramento da qualidade da água para abastecimento público da cidade de Irati/PR. Rev Eng Tecnol. 2014;6(1):92-101.

2. Scorsafava MA, Souza A, Stofer M, Nunes CA, Milanez TV. Avaliação físico-química de água de poços e minas destinadas ao consumo humano. Rev Inst Adolfo Lutz. 2010;69(2):229-32.

3. Joventino ES, Silva SF, Rogério RF, Freitas GL, Ximenes LB, Moura ERF. Comportamento da diarréia infantil antes e após consumo de água pluvialem município do semi-árido brasileiro. Texto \& Contexto Enferm. 2010;19(4):691-9.

4. Araújo GFR, Tonani KAA, Julião FC, Cardoso OO, Alves RIS, Ragazzi MF, et al. Qualidade físico-química e microbiológica da água para consumo humano e a relação com a saúde: estudo em uma comunidade rural no estado de São Paulo. Mundo Saúde. 2011;35(1):98104.

5. Ministério da Saúde (BR). Portaria no 2914 de 12 de dezembro de 2011. Brasília: Ministério da Saúde. 2011 [acesso em 2015 Jan 6]. Disponível em: http:// bvsms.saude.gov.br/bvs/saudelegis/gm/2011/ prt2914_12_12_2011.html

6. Simões TSG, Nunes TCG, Correia BS, Oliveira VPS. Uso de parâmetros físico-químicos e microbiológicos como ferramenta na implementação de projeto de educação ambiental em Cazumbá- São João da Barra, RJ. Bol Observ Amb Alberto Ribeiro Lamego. 2013;7(1):31-48.

7. Ministério da Saúde (BR), Secretaria de Vigilância em Saúde. Inspeção sanitária em abastecimento de água. Brasília: Ministério da Saúde; 2006.
8. Ministério da Saúde (BR). Diretrizes da política nacional de saúde bucal. Brasília: Ministério da Saúde; 2004 [acesso em 2015 Jan 8]. Disponível em: http:// bvsms.saude.gov.br/bvs/politco_nacional_brasil_ sorridente.pdf

9. Bueno RE, Moysés ST, Bueno PAR, Moysés SJ. Determinantes sociais e saúde bucal de adultos nas capitais do Brasil. Rev Panam Salud Publica. 2014;36(1):17-23.

10. Frazão P, Peres MA, Cury JA. Qualidade da água para consumo humano e concentração de fluoreto. Rev Saúde Pública. 2011;45(5):964-73.

11. Peixoto DF, Alencar KP, Peixoto RF, Souza CFM, Sampaio FC, Forte FDS. Heterocontrole da Fluoretação da Água de Abastecimento Público do Município de Jaguaribara, Ceará, Brasil. Rev Bras Promoç Saúde. 2012;25(3):271-7.

12. Medri W, Soeiro JC, Yotsumoto AS, Dalmas JC, Nóbrega MM. Amostragem probabilística no controle da qualidade da água para o consumo humano. Semina. 2012;33(1):49-56.

13. Frazão P, Soares CCS, Fernandes GF, Marques RAA, Narvai PC. Fluoretação da água e insuficiências no sistema de informação da política de vigilância à saúde. Rev Assoc Paul Cir Dent. 2013;67(2):94-100.

14. Bergamo ETP, Barbana M, Terada RSS, Cury JA, Fujimaki M. Fluoride concentrations in the water of Maringá, Brazil, considering the benefit/risk balance of caries and fluorosis. Braz Oral Res. 2015;29(1):1-6

15. Moraes JE, Quináia SP, Takata NH, Fürstenberguer CB. Determinação do índice de fluoreto em águas de abastecimento público em municípios da região centrosul do Paraná. Ambiência. 2009;5(2):233-46.

16. Santos MGC, Santos RC. Fluoretação das Águas de Abastecimento Público no Combate à Cárie Dentária. Rev Bras Ciênc Saúde. 2011;15(1):75-80.

17. Fujibayash SY, ArchettI FB, Pizzatto S, Losso EM, Pizzatto E. Severidade de fluorose dental em um grupo de escolares. RSBO. 2011;8(2):168-73.

18. Instituto Brasileiro de Geografia e estatística (IBGE). Censo Demográfico 2010. [acesso em2015 Jan 10]. Disponível em: http://www.censo2010.ibge.gov.br

19. Programa das Nações Unidas para o Desenvolvimento - PNUD. Atlas do Desenvolvimento Humano no Brasil 2013 [acesso em 2015 Jan 10]. Disponível em: http:// www.atlasbrasil.org.br/2013/pt/ranking 
20. Tejada CO, Jacinto PA, Santos AMA, Sequeira CMD. Mortalidade e as condições socioeconômicas em alagoas. Rev ABET. 2007;7(1):26-51.

21. Alves WA, Correia DS, Barbosa LLB, Lopes LM, Melânia MIASM. Violência letal em Maceió: estudo descritivo sobre homicídios, 2007-2012. Epidemiol Serv Saúde. 2014;23(4):731-40.

22. Cesa K, Abegg C, Aerts D. A Vigilância da fluoretação de águas nas capitais brasileiras. Epidemiol Serv Saúde. 2011;20(4):547-55.

23. Secretaria de Estado da Saúde de Alagoas, Superintendência de Vigilância em Saúde. Relatório Vigiágua 2013: quantidade de municípios que adicionam flúor a água de abastecimento público. Maceió: DIVISAM; 2014.

24. Fundação Nacional de Saúde (BR). Manual de fluoretação da água para consumo humano.Brasília: FUNASA; 2012.

25. Brasil. Decreto No 76.872 de 22 de Dezembro de 1975. Regulamenta a Lei $\mathrm{n}^{\circ} 6.050 / 74$, que dispõe sobre a fluoretação da água. Brasília; 1975.

26. Ministério da Saúde (BR), Secretaria de Vigilância em Saúde. Diretriz Nacional do Plano de Amostragem da Vigilância Ambiental em Saúde Relacionada à Qualidade da Água para Consumo Humano. Brasília: Ministério da Saúde; 2006.

27. Ministério da Saúde (BR), Secretaria de Vigilância em Saúde. Diretriz Nacional do Plano de Amostragem da Vigilância da Qualidade da Água para Consumo
Humano. Brasília: Ministério da Saúde; 2014.

28. Augusto LGS, Gurgel IGD, Câmara-Neto HF, Melo $\mathrm{CH}$, Costa AM. Contexto global e nacional frente aos desafios do acesso adequado à água para consumo humano. Ciênc Saúde Coletiva. 2012;17(6):1511-22.

29. Carmo CDS, Alves CMC, Cavalcante PR, Ribeiro CCC. Avaliação da Fluoretação da água do sistema de abastecimento público na Ilha de São Luis Maranhão, Brasil. Ciênc Saúde Coletiva. 2010;15(Supl 1):183540.

30. Panizzi M, Peres MA. Dez anos de heterocontrole da fluoretação de águas em Chapecó, Estado de Santa Catarina Brasil. Cad Saúde Pública. 2008;24(9):202131.

31. Leivas LL, Tovo MF, Ardenghi TM, Feldens CA, Kramer PF, Faraco Junior IM. Heterocontrole da fluoretação das águas de abastecimento público do Município de Canoas/RS. Stomatos. 2010;16(30):1120.

\section{Endereço de correspondência:}

Guacyra Machado Lisboa

Laboratório Central de Saúde Pública de Alagoas Dr.

Aristeu Lopes - LACEN - AL

Rua Dr. Ernesto Gomes Maranhão, 1773

Bairro: Jatiúca

CEP: 57036-860 - Maceió - AL - Brasil

E-mail: gmlgat@uol.com.br. 